\title{
Molecular Model for the Self-Assembly of the Cyclic Lipodepsipeptide Pseudodesmin A
}

\author{
Jean-Marc Crowet, ${ }^{\dagger}$ Davy Sinnaeve, ${ }^{\S \odot}$ Krisztina Fehér, ${ }^{\perp}$ Yoann Laurin, ${ }^{\dagger}$ Magali Deleu, ${ }^{\dagger}$ \\ José C. Martins, ${ }^{*}+\frac{+}{+}$ and Laurence Lins ${ }^{*} \dagger$ \\ ${ }^{\dagger}$ Laboratory of Molecular Biophysics at Interfaces, TERRA Research Center, Gembloux Agro-Bio Tech, University of Liège, Passage \\ des déportés 2, B-5030 Gembloux, Belgium \\ ${ }^{\S}$ CNRS-Unité de Glycobiologie structurale et fonctionnelle (UGSF) UMR 8576, 50, Avenue de Halley, Campus CNRS de la Haute \\ Borne, 59658 Villeneuve d'Ascq, France \\ ${ }^{\perp}$ Heidelberg Institute for Theoretical Studies, Schloss-Wolfsbrunnenweg 35, 69118 Heidelberg, Germany \\ ${ }^{\ddagger}$ NMR and Structure Analysis Unit, Department of Organic and Macromolecular Chemistry, Ghent University, Krijgslaan 281 S4, \\ B-9000 Gent, Belgium
}

Supporting Information

ABSTRACT: Self-assembly of peptides into supramolecular structures represents an active field of research with potential applications ranging from material science to medicine. Their study typically involves the application of a large toolbox of spectroscopic and imaging techniques. However, quite often, the structural aspects remain underexposed. Besides, molecular modeling of the self-assembly process is usually difficult to handle, since a vast conformational space has to be sampled. Here, we have used an approach that combines short molecular dynamics simulations for peptide dimerization and NMR restraints to build a model of the supramolecular structure from the dimeric units. Experimental NMR data notably provide crucial information about the conformation of the monomeric units, the supramolecular assembly dimensions, and the orientation of the individual peptides within the assembly. This in silico/in vitro mixed approach enables us to define accurate atomistic models of supramolecular structures of the bacterial cyclic lipodepsipeptide pseudodesmin A.

\section{INTRODUCTION}

Resolving the supramolecular structures adopted by short- to medium-sized self-assembling peptide systems is a very challenging task both experimentally and computationally. ${ }^{1-3}$ These structures are of increasing interest due to their potential applications in nanotechnology, biotechnology, and medicine. Supramolecular assemblies with various morphologies find applications such as drug delivery systems, biosensors, imaging contrast agents, antimicrobial agents, or biomaterials for tissue engineering and biomineralization. ${ }^{4-6}$ Peptides offer a biocompatible, easy to use/synthetically accessible, and chemically diverse platform for developing new nanostructures in a controlled fashion. Understanding the forces that drive these building blocks toward self-assembly is of paramount importance for rational design of nanostructures for a specific purpose. Their study by experimental methods typically requires combination of multiple spectroscopic and imaging techniques. ${ }^{4,7-10}$ Higher-order supramolecular structures are hardly amenable to high-resolution approaches such as solution state NMR or X-ray crystallography due to size heterogeneity and/or insolubility.

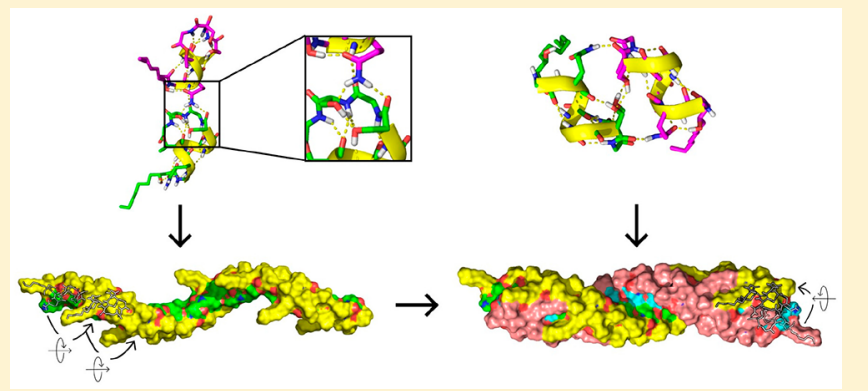
tackle because a high level of sampling of the conformational space is required for the convergence of thermodynamic and structural quantities. All-atom molecular dynamic (MD) simulations have limited ability to reach the long time scales required for the sampling of the molecular degrees of freedom. This issue is typically addressed by employing advanced sampling techniques or by reducing the number of freedom degrees with a coarse grained (CG) representation of molecular entities. ${ }^{2,11,12}$

In this work, we have developed an original approach to circumvent these computational limitations by rationally deducing the supramolecular structures from short MD calculations, guided and validated by NMR experimental data. The system under investigation is the cyclic lipodepsipeptide (CLP) pseudodesmin $\mathrm{A},{ }^{13}$ a pseudomonas secondary metabolite of the viscosin group. ${ }^{14,15}$ Besides interesting biological activity, it displays remarkable self-

Received: August 22, 2019

Revised: September 24, 2019

Published: September 26, 2019 
assembling properties in nonpolar solutions. ${ }^{16-19}$ The precise biological role of the self-assembly is yet to be established for this and other CLPs, although it is speculated that it could occur within the cellular membrane bilayer, which is the apparent target of these compounds for antimicrobial activity. ${ }^{20,21}$ Pseudodesmin A consists of two moieties: (1) a nonapeptide (L-Leu1-D-Gln2-D-aThr3-D-Val4-D-Leu5-D-Ser6-LLeu7-D-Ser8-L-Ile9) cyclized via an ester bond between the Cterminal carboxyl and the D-aThr3 side chain hydroxyl and (2) a fatty acid $((R)-3$-hydroxydecanoic acid) group attached to the $\mathrm{N}$-terminus of the peptide. The monomer conformation was solved by both NMR and X-ray diffraction and features a short left-handed $\alpha$-helix of six amino acids long, followed by a three-residue loop that completes the cyclic structure by covalently stapling the end of the helix to its middle (Figure 1). ${ }^{13}$ The overall molecule is amphipathic, with the hydro-

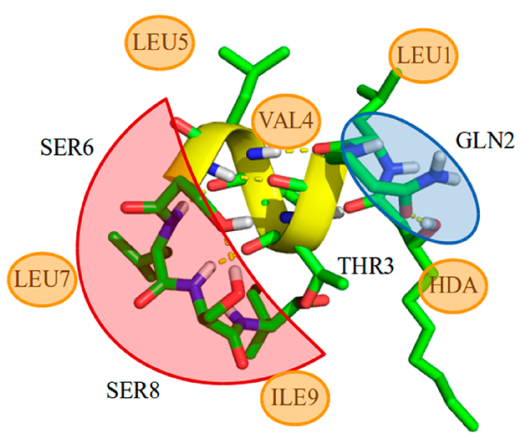

Figure 1. NMR structure of pseudodesmin A. ${ }^{16}$ Carbon hydrogens and backbone carbons of the helix are not represented. The lefthanded helix cartoon and the hydrogen bonds are in yellow. H-bond donors, H-bond acceptors, and hydrophobic amino acids are, respectively, circled in blue, red, and orange.

phobic and hydrophilic residues grouped along opposite sides of the molecular surface. While no self-assembly was observed in acetonitrile solution, the formation of large supramolecular structures has been demonstrated in chloroform. ${ }^{16}$ Using ${ }^{13} \mathrm{C}$ relaxation NMR, it was shown that the assemblies possess an axially symmetric shape and grow along one dimension. ${ }^{17}$ The estimated dimensions suggested that filaments larger than the diameter of one molecule are formed. Also, the orientation of the monomer units relative to the direction of growth can be established, strongly suggesting that the assembly is mediated via intermolecular contacts between free $\mathrm{N}$-terminal amides as hydrogen bond donors and the C-terminal carbonyls as hydrogen bond acceptors. ${ }^{17}$ Intermolecular ROESY (rOe) correlation also confirmed that contacts occur mainly between these parts of the molecule. ${ }^{16}$ However, a detailed atomistic model of the supramolecular assembly remains difficult to establish at this stage. For this reason, we decided to combine in vitro NMR data with molecular modeling.

Our modeling approach involves three steps: (1) scanning of modes of interaction between two monomers by multiple, short MD simulations using intramolecular NMR distance restraints, (2) construction of all feasible supramolecular structures based on the computed dimers, and (3) selection of the correct filament structure based on the experimental ${ }^{13} \mathrm{C}$ NMR relaxation data obtained in Sinnaeve et al. 2012. ${ }^{17}$ The complementarity of MD simulations and NMR data is crucial here, since each separate technique (in silico and in vitro) is not sufficient to obtain an accurate atomistic model.

\section{METHODS}

Atomistic Molecular Dynamics. Simulations have been performed with the GROMOS 54a7 force field. ${ }^{22}$ The NMR structure of pseudodesmin (L-Leu1-D-Gln2-D-aThr3-D-Val4-DLeu5-D-Ser6-L-Leu7-D-Ser8-L-Ile9; Figure 1) has been used. ${ }^{13}$ The D-amino acids and the allo-threonine have been added to the force field by adapting the improper dihedral definition of the alpha and beta carbons, respectively. From L-amino acid topologies, the improper dihedrals are inverted at the C-alpha to model D-amino acids. HDA is derived from threonine, and parameters for ester cyclization have been derived from ethyl acetate. $^{22}$ The model used to simulate chloroform is the one present natively in the force field, and acetonitrile parameters are coming from the ATB repository. ${ }^{23}$ rOe distance restraints defined in Sinnaeve et al. $2009^{16}$ (Table S1) were used during the molecular dynamics simulations. As the carbon hydrogens are not represented in the Gromos force fields, $0.1 \mathrm{~nm}$ was added to the distance restraints in these cases. All of the systems studied were first minimized by steepest descent for 5000 steps. They were then run for a $1 \mathrm{~ns}$ simulation with the peptide under position restraints in periodic boundary conditions (PBCs) using a 2 fs time step. Production runs were performed for $50 \mathrm{~ns}$. All of the systems were solvated with SPC water, ${ }^{24}$ chloroform, or acetonitrile. The dynamics were carried out under the NPT conditions (298 K and 1 bar). Temperature was maintained by using the v-rescale method ${ }^{25}$ with $\tau_{\mathrm{T}}=0.2 \mathrm{ps}$, and an isotropic pressure was maintained by using the Parrinello-Rahman ${ }^{26}$ barostat with a compressibility of $4.5 \times 10^{5}(1 / \mathrm{bar})$ and $\tau_{\mathrm{P}}=1$ ps. Electrostatic interactions were treated by using the particle mesh Ewald (PME) method. ${ }^{27}$ van der Waals and electrostatics were treated with a $1.0 \mathrm{~nm}$ cutoff. Bond lengths were maintained with the LINCS $^{28}$ algorithm. The trajectories were performed and analyzed with the GROMACS 4.5.4 tools as well as with homemade scripts and software, and 3D structures were analyzed with both PYMOL ${ }^{29}$ and $\mathrm{VMD}^{30}$ software.

\section{RESULTS}

Simulation of Solvent Dependent Self-Assembly: Acetonitrile versus Chloroform. In order to reproduce the behavior of the peptides observed experimentally in both acetonitrile (no self-assembly observed) and chloroform (strong self-assembly), we performed short standard MD simulations in these solvents. It has already been established by NMR that the peptide monomer adopts essentially the same intramolecular conformation in both acetonitrile and chloroform. ${ }^{16}$ In order to reduce the degrees of motional freedom, intramolecular NMR distance restraints (Table S1) obtained from acetonitrile solution are used here to restrain the peptide structure during the simulations to avoid drifting too far from the experimental conformation. Simulation of four peptides in an acetonitrile solvent box for $250 \mathrm{~ns}$ showed that peptides stay mainly monomeric, in agreement with experimental data. ${ }^{16}$ Dimers can be formed transiently but represent less than $13.5 \%$ of the simulation time. In contrast, in chloroform, peptides rapidly interact with each other and form multimers during the whole simulation time (Figure S1). However, due to the many possible modes of intermolecular interaction between the peptides, using a brute-force approach to sample a larger number of peptides in a large solvent box in order to observe the formation of a well-defined supramolecular 
A

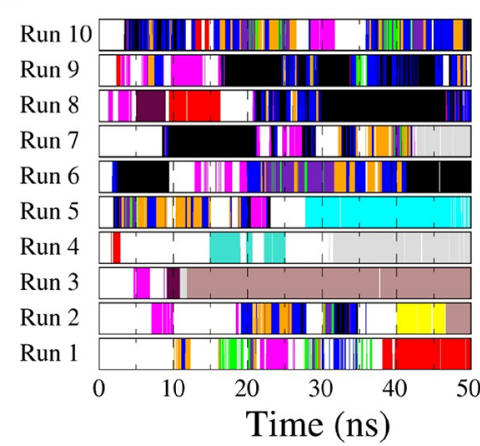

B

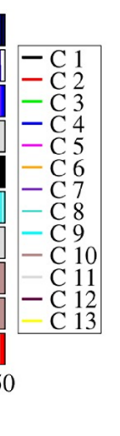

Cluster 1

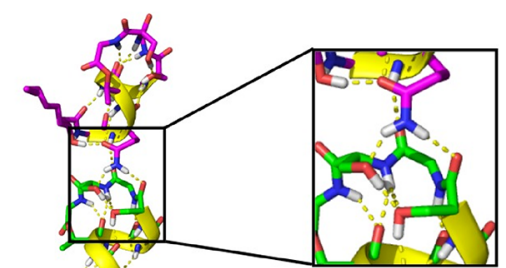

$\sqrt{\mathrm{s} g}$
Cluster 9

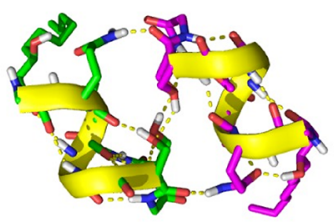

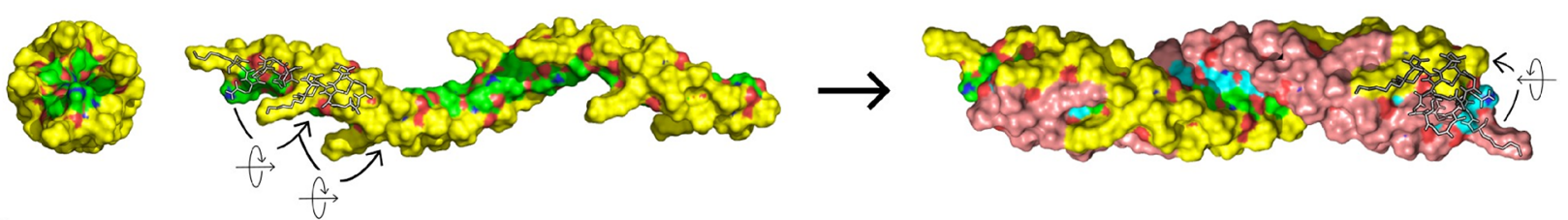

C

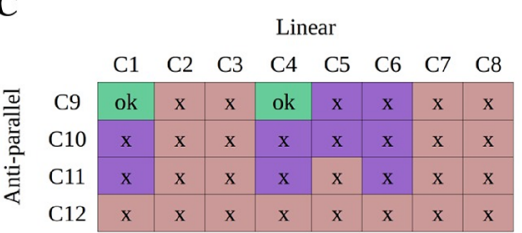

D

Clusters 4 and 10

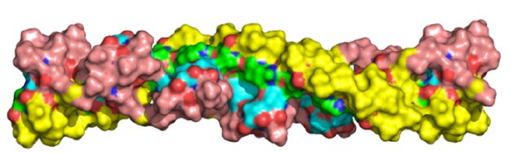

Clusters 3 and 11

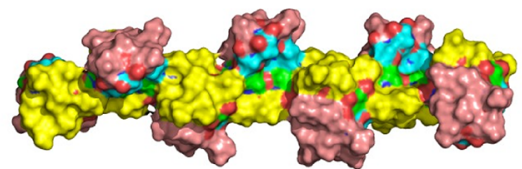

Figure 2. Cluster occurrence and filament construction. (A) Cluster occurrence in each simulation run. (B) Representation of the dimer from clusters $\mathrm{C} 1$ and $\mathrm{C} 9$ and their association to build the filaments. Carbon hydrogens and backbone carbons of the helix are not represented. The lefthanded helix cartoon and the hydrogen bonds are in yellow. The filaments built from clusters C1 and C9 are drawn with a surface representation. Hydrophobic residues are represented in yellow and pink, while hydrophilic residues are represented in green and blue. (C) Summary table of the pseudodesmin association; pink means no second filament is formed, i.e., molecules do not align (Figure 2D, right); purple, the second filament forms, but the molecules are not aligned correctly (Figure 2D, left); and green, the second filament forms in the correct way. (D) Examples of second filaments not correctly formed.

Table 1. H-Bonds Defining Each Cluster ${ }^{a}$

\begin{tabular}{|c|c|c|c|c|c|}
\hline cluster & relative orientation & hydrogen bond criterion & cluster & relative orientation & hydrogen bond criterion \\
\hline \multirow[t]{2}{*}{ cluster 1} & linear & GLN2 NH-LEU7 CO & cluster 8 & linear & HDA OH-VAL4 CO \\
\hline & & GLN2 $\mathrm{NH}_{2} E$-SER6 CO & & & LEU1 NH-LEU5 CO \\
\hline \multirow[t]{2}{*}{ cluster 2} & linear & LEU1 NH-LEU7 CO & & & GLN2 NH-SER6 CO \\
\hline & & GLN2 $\mathrm{NH}_{2} Z$-SER8 CO & & & GLN2 $\mathrm{NH}_{2} E$-LEU7 CO \\
\hline \multirow[t]{2}{*}{ cluster 3} & linear & LEU1 NH-SER6 CO & cluster 9 & antiparallel & GLN2 $\mathrm{NH}_{2} E$-SER6 CO \\
\hline & & GLN2 $\mathrm{NH}_{2} Z$-LEU5 $\mathrm{CO}$ & & & SER6 CO-GLN2 $\mathrm{NH}_{2} E$ \\
\hline \multirow[t]{2}{*}{ cluster 4} & linear & LEU1 NH-LEU7 CO & cluster 10 & antiparallel & GLN2 $\mathrm{NH}_{2} E$-SER8 OH \\
\hline & & GLN2 $\mathrm{NH}_{2} Z$-SER6 CO & & & SER8 OH-GLN2 $\mathrm{NH}_{2} E$ \\
\hline \multirow[t]{2}{*}{ cluster 5} & linear & LEU1 NH-SER8 CO & cluster 11 & antiparallel & GLN2 $\mathrm{NH}_{2} E$-SER8 CO \\
\hline & & GLN2 $\mathrm{NH}_{2} Z$-LEU7 CO & & & SER6 CO-GLN2 $\mathrm{NH}_{2} E$ \\
\hline \multirow[t]{3}{*}{ cluster 6} & linear & HDA OH-SER6 CO & cluster 12 & antiparallel & GLN2 $\mathrm{NH}_{2} E$-LEU5 $\mathrm{CO}$ \\
\hline & & LEU1 NH-LEU7 CO & & & \\
\hline & & GLN2 $\mathrm{NH}_{2} Z$-LEU5 CO & & & SER8 CO-GLN2 $\mathrm{NH}_{2} E$ \\
\hline \multirow[t]{3}{*}{ cluster 7} & linear & HDA OH-LEUS CO & cluster 13 & perpendicular & GLN2 NH-SER8 CO \\
\hline & & LEU1 NH-SER6 CO & & & GLN2 $\mathrm{NH}_{2} E$-SER8 OH \\
\hline & & GLN2 $\mathrm{NH}_{2} Z$-VAL4 CO & & & GLN2 $\mathrm{NH}_{2} \mathrm{Z}-\mathrm{THR} 3 \mathrm{OT}$ \\
\hline
\end{tabular}

${ }^{a} \mathrm{NH}_{2} \mathrm{Z}$ corresponds to the $\mathrm{H}$ which is on the $\mathrm{O}$ side of the planar amide bond (Figure $2 \mathrm{~B}$ ). OT corresponds to the oxygen of the alkoxy group.

structure is unlikely to succeed within a reasonable period of time using currently available computational power.

Dimer Scan. We chose an alternative approach where we first scan all possible interactions between only two peptides in chloroform. For this, 10 independent simulations of $50 \mathrm{~ns}$ were carried out (Figure 2A). Those simulations showed that intermolecular contacts arise mainly through hydrogen bond formation between the $\mathrm{N}$-terminal of one peptide and the $\mathrm{C}$ - terminal residue of the other, as shown in Figure $2 \mathrm{~B}$ and in agreement with what was already proposed via NMR. ${ }^{16}$ Several possible transient intermolecular hydrogen bonds are observed during the simulation time, and most often several hydrogen bonds are formed simultaneously (Table S2). Hydrogen bonding patterns that are associated with specific dimeric organizations can be identified and can be used as a criterion to define dimer structures (Figure S2). Thirteen clusters were 
hence classified, each representing at least $0.5 \%$ of the total simulation time. Their hydrogen bonds are listed in Tables 1 and S2 and represented in Figures $2 \mathrm{~A}$ and S2. Three main types of clusters are observed based on the relative orientation of the peptides: (1) both peptides stacked linearly upon each other via their N- and C-terminal sides (Figure 2B, left); (2) both peptides aggregated side-by-side in an antiparallel fashion (Figure 2B, right); (3) both peptides oriented in a perpendicular arrangement for one cluster (C13) (Table 1 and Figure S3). The clusters showing the longer time duration are the antiparallel dimers $\mathrm{C} 9-\mathrm{C} 11$ and the two linear clusters $\mathrm{C} 1$ and $\mathrm{C} 2$ (Table 1).

Construction of One Filament Supramolecular Structures. In the linear dimers, the peptides interact via the hydrogen bond donor side of one molecule and the acceptor side of the other one (cluster C1 of the Figure 2B) and can then lead to the oligomerization of the peptides. Filamentous oligomers have been built by iterative translations and rotations (Figure 2B). First, from one member of the dimers, translations and rotations necessary to find the coordinates of the second member of the dimer are computed (Figure 2B). From this transformation matrix, filamentous oligomers that retain the dimer structure and intermolecular contacts are built. For example, the filament formed from cluster $\mathrm{C} 1$ shows a helically twisted structure with five peptides per turn and all of the hydrophilic residues facing inward (Figure 2). Different results are obtained from the various other linear clusters, as summarized in Figure S4.

Validation of Filamentous Structures Based on NMR

Data. Experimental NMR results provided the orientation of the monomer within the supramolecular structure relative to the direction of growth. ${ }^{17}$ This information can be directly confronted with the filaments obtained by molecular modeling. This orientation is reflected in the collection of angles between various $\mathrm{C} \alpha-\mathrm{H} \alpha$ bonds of each amino acid and the direction of growth (long axis) of the supramolecular structure, as this is what was obtained from the NMR ${ }^{13} \mathrm{C}$ relaxation approach. ${ }^{17}$ The clusters giving filament models displaying the best correlation with experimental data are clusters C1, C2, C4, and C5 with average differences over 10 angles of 13.5, 10, 10, and $13.6^{\circ}$ respectively, while the cluster displaying the greatest differences is cluster $\mathrm{C} 7$ with an average difference of $20.6^{\circ}$ (Figure 3, Table S3).

Construction of the Two-Stranded Filament Structures. The segregation of hydrophobic and hydrophilic residues along some filaments (see, for example, Figure 2B, left, or Figure S4, cluster C1 or C4) strongly suggests that pseudodesmin A can form intertwisted filaments by association of the hydrophilic residues. Clusters $\mathrm{C} 1$ and $\mathrm{C} 4$ with five and seven peptides per turn are very good candidates, while the other filaments have four or less peptides per turn, with some hydrophilic residues facing outside, and should then be less prone to self-assemble. To build the second filament, the antiparallel dimers have been used as a template. The translations and rotations necessary to go from one member of the antiparallel dimer to the other are computed and applied to each peptide of the linear filament. The eight linear filament models and the four antiparallel dimers have been used to build double-stranded filaments (Figure S5). When we look at Table 1, one can notice that the hydrogen bonds defining linear and antiparallel dimers both involve the side chain of GLN2. It supposes that the conformation of the side chain of GLN2 is different in the two structures used to build double-

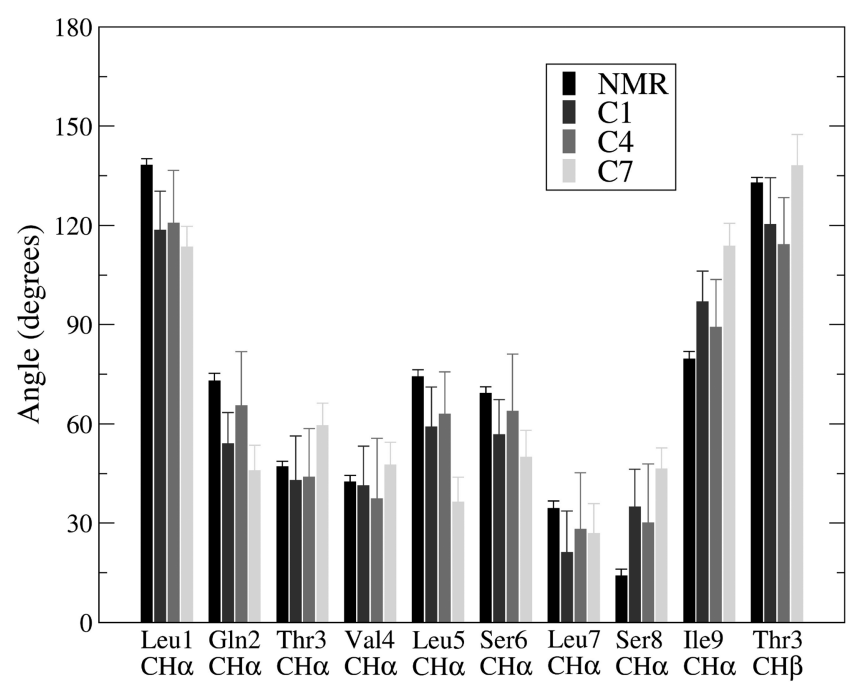

Figure 3. Correlation between $\mathrm{NMR}{ }^{13} \mathrm{C}$ relaxation data and $\mathrm{C} 1, \mathrm{C} 4$, or $\mathrm{C} 7$ structures about the angles between $\mathrm{C} \alpha \mathrm{H}$ and the long axis of the filament for each residue.

stranded filaments, as clearly illustrated in Figure 2B for C1 and C9. Thus, to build a double filament, the conformation of the side chain of GLN2 from the linear dimer has been kept, while the hydrogen bonds formed between the hydroxyl groups of SER6 and SER8 of each peptide in the antiparallel configuration (Figures $2 \mathrm{~B}$ and S3) have been conserved. When looking at the antiparallel clusters, C9 is the one where the hydrophilic faces are matching at best (Figure S3), and from the 32 possible double-stranded filaments built, two combinations $(\mathrm{C} 1+9$ and $\mathrm{C} 4+9)$ give a second filament which is structurally identical to the one-strand filament and are thus the best candidates. It is worth noting that, from two unrelated dimer configurations (linear and antiparallel), a structure with two structurally identical filaments can be built, strongly suggesting self-consistency of the approach. Besides, we can notice in Figure $2 \mathrm{~A}$ that $\mathrm{C} 1$ and $\mathrm{C} 4$ are frequently observed concomitantly during the simulations, while $\mathrm{C} 1$ appears to be more stable over time. Since C9 is present during almost all of the simulation time (Figure $2 \mathrm{~A}$ ), we can assume that the two double filament structures could exist as "metastable" conformations that can switch from one to the other.

To further validate our filament models, intermolecular ROESY correlations measured by NMR on the aggregates ${ }^{16}$ highlighting molecular contacts can be compared to those observed between the peptides inside the model filament structures. From Table 2 listing the interatomic distances computed on the cluster models, it is obvious that almost all contacts can be explained by the formation of one or the other linear filaments, with clusters $\mathrm{C} 1$ and $\mathrm{C} 2$ presenting notably seven interatomic distances lower than $5 \AA$ A. Regarding the other filament candidate made from clusters $\mathrm{C} 4$ and $\mathrm{C} 9$, it can explain five of the interatomic distances and notably the contact between LEU1 and LEU7 which is not observed in the $\mathrm{C} 1+9$ double filaments. We can also notice that the antiparallel configurations reflect the proximity between the side chain of GLN2 and the backbone of SER8. Its proximity with the ILE9 residue is less obvious. With the latter being in the middle of the hydrophobic face of the peptide, no cluster presents interatomic distances smaller than $5 \AA$, except in cluster $\mathrm{C} 13$, which is however in a perpendicular configuration. Finally, we compared the diameter measured on the two- 
Table 2. List of the Atomic Contacts Determined Experimentally by NMR and the Interatomic Distance Computed in Each Model Dimer Cluster ${ }^{a}$

\begin{tabular}{|c|c|c|c|c|c|c|c|c|c|c|c|c|c|}
\hline & $\mathrm{C} 1$ & $\mathrm{C} 2$ & C3 & $\mathrm{C} 4$ & $\mathrm{C} 5$ & C6 & $\mathrm{C} 7$ & $\mathrm{C} 8$ & $\mathrm{C} 9$ & $\mathrm{C} 10$ & $\mathrm{C} 11$ & $\mathrm{C} 12$ & $\mathrm{C} 13$ \\
\hline HDA HA-SER8 HA & 4.6 & 5.0 & 10.4 & 3.6 & 4.5 & 4.2 & 8.3 & 10.3 & 9.8 & 11.0 & 6.9 & 9.8 & 8.4 \\
\hline HDA OH-SER8 HA & 3.2 & 3.8 & 9.0 & 4.8 & 3.3 & 5.7 & 9.0 & 7.7 & 7.7 & 7.9 & 3.9 & 7.5 & 6.9 \\
\hline LEU1 HN-LEU7 HA & 6.4 & 4.0 & 5.4 & 3.3 & 7.9 & 2.9 & 3.2 & 5.0 & 10.5 & 13.1 & 9.6 & 10.8 & 9.6 \\
\hline LEU1 HN-SER8 HA & 2.7 & 3.1 & 7.8 & 3.8 & 3.7 & 4.4 & 6.6 & 8.7 & 10.4 & 9.4 & 5.9 & 7.3 & 5.9 \\
\hline LEU1 HN-ILE9 HB & 6.9 & 8.0 & 12.2 & 8.7 & 5.9 & 9.6 & 10.9 & 11.7 & 16.3 & 9.8 & 9.9 & 11.3 & 4.6 \\
\hline GLN2 HN-LEU7 HA & 4.1 & 7.0 & 3.8 & 3.9 & 7.7 & 4.0 & 4.2 & 3.3 & 8.1 & 11.0 & 7.9 & 9.2 & 8.8 \\
\hline GLN2 HN-SER8 HA & 3.1 & 4.0 & 7.3 & 6.3 & 4.5 & 7.2 & 8.5 & 6.7 & 8.6 & 7.9 & 5.7 & 5.5 & 4.6 \\
\hline GLN2 HN-ILE9 HB & 8.7 & 8.5 & 11.5 & 11.0 & 7.0 & 11.8 & 11.6 & 11.1 & 14.1 & 9.5 & 8.3 & 8.2 & 6.6 \\
\hline GLN2 $\mathrm{NH}_{2} E$-SER8 HA & 3.9 & 4.9 & 8.5 & 8.2 & 4.4 & 9.4 & 9.6 & 3.6 & 4.9 & 4.4 & 4.3 & 4.0 & 4.5 \\
\hline GLN2 $\mathrm{NH}_{2} Z$-SER8 HA & 2.8 & 3.5 & 8.4 & 7.1 & 2.8 & 7.9 & 8.3 & 4.5 & 4.4 & 4.9 & 3.6 & 4.8 & 5.2 \\
\hline
\end{tabular}

stranded filament models $\mathrm{C} 1+9$ and $\mathrm{C} 4+9(2.1$ and $2.3 \mathrm{~nm}$, respectively) to the diameter of the aggregate measured by NMR $(2.5 \mathrm{~nm}),{ }^{19}$ showing a very good agreement. On the other hand, the length of aggregates measured by NMR is 6.4 $\mathrm{nm},{ }^{17}$ corresponding to structures with an average of five and four peptides per filament in the $\mathrm{C} 1+9(6.4 \mathrm{~nm})$ and $\mathrm{C} 4+9$ $(6.2 \mathrm{~nm})$ models, respectively. Overall, there is a very good agreement between the proposed models for double-stranded filaments and the NMR data, strongly supporting our modeling approach.

\section{DISCUSSION}

The description of peptide self-assembly in order to reach molecular models at atomistic level remains a nontrivial and nonobvious task both experimentally and computationally but is of paramount importance for the rational design of nanostructures with specific purposes. To study the peptide self-assembly by experimental methods, it typically requires combination of multiple spectroscopic (NMR, FTIR, CD spectroscopy, ...) and imaging techniques (TEM, AFM, ...) because supramolecular structures are less amenable to highresolution approaches such as solution state NMR or X-ray crystallography. ${ }^{31}$ The peptide under investigation in this study, pseudodesmin A, has been the object of an in depth experimental characterization through various NMR and X-ray diffraction techniques. ${ }^{13,16,17}$ Experimental NMR data obtained in chloroform notably provide significant information about the conformation of the monomeric units, the supramolecular assembly dimensions, and the orientation and intermolecular contacts of the individual peptides within the assembly. ${ }^{16,17}$ However, while very informative, these data were not sufficient to reach an atomistic representation of the aggregates.

Here, we have used an original approach that combines short molecular dynamics simulations of the dimers and NMR restraints to build a model of the supramolecular structure from the dimeric units. The first step of the approach is to calculate dimers of the peptide based on its restrained NMR monomer structure by multiple short MD simulations. Sampling dimer formation and hydrogen bond patterning led us to classify the dimers into 13 clusters in which intermolecular contacts arise mainly through hydrogen bond formation between the N-terminal part of one peptide and the C-terminal residue of the other, in agreement with NMR data. ${ }^{16}$ The dimers can either be linear or antiparallel. More precisely, we found that the interactions and peptide configurations observed for the linear cluster $\mathrm{C} 1$ are very favorable to filament formation, according to NMR data.
Moreover, this filament presents a partition of its hydrophobic and hydrophilic residue that allows the formation of complementary and identical filaments, when combined with the antiparallel cluster C9. In addition, these two clusters are the ones that are more populated during molecular dynamics simulations. Another potential structure is made of C9 and C4 clusters, which presents these same properties. Both oligomeric structures converge, strongly supporting our methodology. The oligomeric structure further appears to fit all of the available experimental information (intermolecular contacts, orientation, shape, and size) and provides now a working model of the pseudodesmin self-assembly.

It should be noted that simulating self-assembly processes by $\mathrm{MD}$ is not an obvious task and all-atom molecular dynamics (AT-MD) simulations have limited ability to reach the long time scales required for the sampling of the molecular degrees of freedom associated with the self-assembly. ${ }^{32,33}$ The formation of small oligomers, the earliest stages of the selfassembly, can be performed by AT-MD, usually with advanced sampling techniques such as replica exchange molecular dynamics (REMD). ${ }^{32,34-38}$ Multiscale approaches using REMD and an implicit water force field such as OPEP coupled to all-atom MD simulations notably allowed simulation of the self-organization of Sup35 amyloid peptides without preformed seeds. ${ }^{12}$ In the same way, activationrelaxation technique (ART) simulations allowing the energy landscape to be sampled were coupled to coarse grained energy models and AT-MD simulations to study the early steps of amyloid peptide aggregation. ${ }^{39}$ These advanced techniques are required to sample the conformational space of monomers to find the ones involved in the oligomers. In our study, the high stability of the peptide conformation as a monomer and in aggregates allows us, by using NMR restraints on monomers, to directly sample the dimerization processes by using short AT-MD simulations. MD also allows simulation of the dimerization in the same environment experimentally (chloroform or acetonitrile). For the formation of larger oligomers, coarse grained (CG) representations, which reduce the number of freedom degrees, have been successfully applied. CG representations have notably been used to simulate the formation of nanostructures, ${ }^{2,40,41}$ fibrils of $\mathrm{A} \beta$ peptide, ${ }^{35}$ or cylindrical micelle fiber of peptide amphiphiles. ${ }^{42}$ However, CG-MD simulations are not always sufficiently accurate to study self-assembly. In our study, CG representation like MARTINI cannot be used due to the limitation of this method in reproducing hydrogen bond interactions. ${ }^{43}$ Indeed, this peptide has a hydrogen bond donor side and an acceptor side 
that could not be distinguished in a CG representation. To address self-assembly in this context, the well-defined dimer structures identified during the dimerization simulation enabled the exploration of larger oligomer structures and allowed the identification of accurate model filament candidates. To study the later stage of self-assembly, model aggregates are usually preformed prior to AT-MD simulations, such as preformed amyloid fibrils built by stacking beta strands ${ }^{44-47}$ or peptides assembled into cylindrical nanofibers. ${ }^{48,49}$ In contrast to pseudodesmin A, these peptides do not have a well-defined conformation and present a wide range of interactions inside the aggregates. ${ }^{9,10,50}$ A way to solve this problem is to validate these interactions against experimental information like intermolecular contacts identified by NMR., ${ }^{9,52}$ In our study, experimental observations also help to build and validate the aggregate structure obtained by simulations. The complementarity of $\mathrm{MD}$ simulations and NMR data is crucial here, since each separate technique (in silico and in vitro) was not sufficient to obtain an accurate atomistic model.

\section{CONCLUSIONS}

In conclusion, we have used a fast $\mathrm{MD}$ and modeling approach to obtain supramolecular structures at the atomistic level, since sampling of the dimers is made with short simulation times. Model filaments can be built from the computed dimer structures, by using NMR data to guide and validate atomistic models step by step. Our original in silico/in vitro approach has allowed solving a harsh structural problem, i.e., peptide selfassembly for a cyclic aggregating peptide, pseudodesmin A. This stumbling block would not have been solved by CG-MD methods or AT-MD brute force only. The proposed strategy could hence be applied to other aggregating peptides having a conformational stability as monomer or dimer and could be used for rational design of nanostructures for specific purposes.

\section{ASSOCIATED CONTENT}

\section{S Supporting Information}

The Supporting Information is available free of charge on the ACS Publications website at DOI: 10.1021/acs.jpcb.9b08035.

Table S1, distance restraints from NMR used during simulations; Table S2, H-bonds defining each cluster; Table S3, correlation between NMR ${ }^{13} \mathrm{C}$ relaxation and the angles between $\mathrm{C} \alpha \mathrm{H}$ and the long axis of filaments made from each cluster; Figure S1, distance from pseudodesmin mass center during simulations in acetonitrile and chloroform; Figure S2, RMSD between the central structure of each cluster and all other dimeric structures; Figure S3, representation of the dimers; Figure S4, filaments built from linear clusters; Figure S5, filaments built from pairs of linear and antiparallel clusters (PDF)

\section{AUTHOR INFORMATION}

\section{Corresponding Authors}

*E-mail: 1.lins@uliege.be (L.L.).

*E-mail: Jose.Martins@UGent.be (J.C.M.).

\section{ORCID $\odot$}

Jean-Marc Crowet: 0000-0002-9252-2396

Davy Sinnaeve: 0000-0003-2556-5895

Magali Deleu: 0000-0001-8255-2965

\section{Notes}

The authors declare no competing financial interest.

\section{ACKNOWLEDGMENTS}

L.L. and M.D. thank the FRS-FNRS for financial support. D.S. thanks the Research Foundation-Flanders for a postdoctoral fellowship. This work is supported by the FRS-FNRS (BRIDGING CDR J.0114.18 and RHAMEMB CDR J.0086.18, PDR T.0063.19). Partial computational resources have been provided by the Consortium des Équipements de Calcul Intensif (CÉCI), funded by the Fonds de la Recherche Scientifique de Belgique (F.R.S.-FNRS) under Grant No. 2.5020.11.

\section{REFERENCES}

(1) Colombo, G.; Soto, P.; Gazit, E. Peptide Self-Assembly at the Nanoscale: A Challenging Target for Computational and Experimental Biotechnology. Trends Biotechnol. 2007, 25 (5), 211-218.

(2) Frederix, P. W. J. M.; Patmanidis, I.; Marrink, S. J. Molecular Simulations of Self-Assembling Bio-Inspired Supramolecular Systems and Their Connection to Experiments. Chem. Soc. Rev. 2018, 47 (10), 3470-3489.

(3) Tuttle, T. Computational Approaches to Understanding the SelfAssembly of Peptide-Based Nanostructures. Isr. J. Chem. 2015, 55 (67), 724-734.

(4) Mandal, D.; Nasrolahi Shirazi, A.; Parang, K. Self-Assembly of Peptides to Nanostructures. Org. Biomol. Chem. 2014, 12 (22), 35443561.

(5) Qiu, F.; Chen, Y.; Tang, C.; Zhao, X. Amphiphilic Peptides as Novel Nanomaterials: Design, Self-Assembly and Application. Int. J. Nanomed. 2018, 13, 5003-5022.

(6) Eskandari, S.; Guerin, T.; Toth, I.; Stephenson, R. J. Recent Advances in Self-Assembled Peptides: Implications for Targeted Drug Delivery and Vaccine Engineering. Adv. Drug Delivery Rev. 2017, 110-111, 169-187.

(7) Nieuwland, M.; Ruizendaal, L.; Brinkmann, A.; KroonBatenburg, L.; van Hest, J. C. M.; Löwik, D. W. P. M. A Structural Study of the Self-Assembly of a Palmitoyl Peptide Amphiphile. Faraday Discuss. 2013, 166 (0), 361.

(8) Hollamby, M. J.; Karny, M.; Bomans, P. H. H.; Sommerdjik, N. A. J. M.; Saeki, A.; Seki, S.; Minamikawa, H.; Grillo, I.; Pauw, B. R.; Brown, P.; et al. Directed Assembly of Optoelectronically Active Alkyl- $\pi$-Conjugated Molecules by Adding n-Alkanes or $\pi$-Conjugated Species. Nat. Chem. 2014, 6 (8), 690-696.

(9) Rad-Malekshahi, M.; Visscher, K. M.; Rodrigues, J. P. G. L. M.; de Vries, R.; Hennink, W. E.; Baldus, M.; Bonvin, A. M. J. J.; Mastrobattista, E.; Weingarth, M. The Supramolecular Organization of a Peptide-Based Nanocarrier at High Molecular Detail. J. Am. Chem. Soc. 2015, 137 (24), 7775-7784.

(10) Yu, Z.; Erbas, A.; Tantakitti, F.; Palmer, L. C.; Jackman, J. A.; Olvera de la Cruz, M.; Cho, N.-J.; Stupp, S. I. Co-Assembly of Peptide Amphiphiles and Lipids into Supramolecular Nanostructures Driven by Anion- $\pi$ Interactions. J. Am. Chem. Soc. 2017, 139 (23), 78237830.

(11) Yuan, C.; Li, S.; Zou, Q.; Ren, Y.; Yan, X. Multiscale Simulations for Understanding the Evolution and Mechanism of Hierarchical Peptide Self-Assembly. Phys. Chem. Chem. Phys. 2017, 19 (35), 23614-23631.

(12) Nasica-Labouze, J.; Meli, M.; Derreumaux, P.; Colombo, G.; Mousseau, N. A Multiscale Approach to Characterize the Early Aggregation Steps of the Amyloid-Forming Peptide GNNQQNY from the Yeast Prion Sup-35. PLoS Comput. Biol. 2011, 7 (5), No. e1002051.

(13) Sinnaeve, D.; Michaux, C.; Van hemel, J.; Vandenkerckhove, J.; Peys, E.; Borremans, F. a. M.; Sas, B.; Wouters, J.; Martins, J. C. Structure and X-Ray Conformation of Pseudodesmins A and B, Two 
New Cyclic Lipodepsipeptides from Pseudomonas Bacteria. Tetrahedron 2009, 65 (21), 4173-4181.

(14) Raaijmakers, J. M.; de Bruijn, I.; de Kock, M. J. D. Cyclic Lipopeptide Production by Plant-Associated Pseudomonas Spp.: Diversity, Activity, Biosynthesis, and Regulation. Mol. Plant-Microbe Interact. 2006, 19 (7), 699-710.

(15) Gross, H.; Loper, J. E. Genomics of Secondary Metabolite Production by Pseudomonas Spp. Nat. Prod. Rep. 2009, 26 (11), 1408.

(16) Sinnaeve, D.; Hendrickx, P. M. S.; Van Hemel, J.; Peys, E.; Kieffer, B.; Martins, J. C. The Solution Structure and Self-Association Properties of the Cyclic Lipodepsipeptide Pseudodesmin A Support Its Pore-Forming Potential. Chem. - Eur. J. 2009, 15 (46), 1265312662.

(17) Sinnaeve, D.; Delsuc, M.-A.; Martins, J. C.; Kieffer, B. Insight into Peptide Self-Assembly from Anisotropic Rotational Diffusion Derived from 13C NMR Relaxation. Chem. Sci. 2012, 3 (4), 1284.

(18) De Vleeschouwer, M.; Sinnaeve, D.; Van den Begin, J.; Coenye, T.; Martins, J. C.; Madder, A. Rapid Total Synthesis of Cyclic Lipodepsipeptides as a Premise to Investigate Their Self-Assembly and Biological Activity. Chem. - Eur. J. 2014, 20 (25), 7766-7775.

(19) Geudens, N.; De Vleeschouwer, M.; Fehér, K.; Rokni-Zadeh, H.; Ghequire, M. G. K.; Madder, A.; De Mot, R.; Martins, J. C.; Sinnaeve, D. Impact of a Stereocentre Inversion in Cyclic Lipodepsipeptides from the Viscosin Group: A Comparative Study of the Viscosinamide and Pseudodesmin Conformation and Self-Assembly. ChemBioChem 2014, 15 (18), 2736-2746.

(20) Geudens, N.; Nasir, M. N.; Crowet, J.-M.; Raaijmakers, J. M.; Fehér, K.; Coenye, T.; Martins, J. C.; Lins, L.; Sinnaeve, D.; Deleu, M. Membrane Interactions of Natural Cyclic Lipodepsipeptides of the Viscosin Group. Biochim. Biophys. Acta, Biomembr. 2017, 1859 (3), 331-339.

(21) Khavani, M.; Izadyar, M.; Housaindokht, M. R. Theoretical Design of the Cyclic Lipopeptide Nanotube as a Molecular Channel in the Lipid Bilayer, Molecular Dynamics and Quantum Mechanics Approach. Phys. Chem. Chem. Phys. 2015, 17 (38), 25536-25549.

(22) Schmid, N.; Eichenberger, A. P.; Choutko, A.; Riniker, S.; Winger, M.; Mark, A. E.; van Gunsteren, W. F. Definition and Testing of the GROMOS Force-Field Versions 54A7 and 54B7. Eur. Biophys. J. 2011, 40 (7), 843-856.

(23) Malde, A. K.; Zuo, L.; Breeze, M.; Stroet, M.; Poger, D.; Nair, P. C.; Oostenbrink, C.; Mark, A. E. An Automated Force Field Topology Builder (ATB) and Repository: Version 1.0. J. Chem. Theory Comput. 2011, 7 (12), 4026-4037.

(24) Hermans, J.; Berendsen, H. J. C.; Van Gunsteren, W. F.; Postma, J. P. M. A Consistent Empirical Potential for Water-Protein Interactions. Biopolymers 1984, 23 (8), 1513-1518.

(25) Bussi, G.; Donadio, D.; Parrinello, M. Canonical Sampling through Velocity Rescaling. J. Chem. Phys. 2007, 126 (1), 014101.

(26) Parrinello, M. Polymorphic Transitions in Single Crystals: A New Molecular Dynamics Method. J. Appl. Phys. 1981, 52 (12), 7182.

(27) Essmann, U.; Perera, L.; Berkowitz, M. L.; Darden, T.; Lee, H.; Pedersen, L. G. A Smooth Particle Mesh Ewald Method. J. Chem. Phys. 1995, 103 (19), 8577.

(28) Hess, B.; Bekker, H.; Berendsen, H. J. C.; Fraaije, J. G. E. M. LINCS: A Linear Constraint Solver for Molecular Simulations. J. Comput. Chem. 1997, 18 (12), 1463-1472.

(29) Schrödinger, L. L. C. The PyMOL Molecular Graphics System, Version 1.3. Methods Enzymol. 2010, 266, 540-553.

(30) Humphrey, W.; Dalke, A.; Schulten, K. VMD: Visual Molecular Dynamics. J. Mol. Graphics 1996, 14 (1), 33-38.

(31) Ekiz, M. S.; Cinar, G.; Khalily, M. A.; Guler, M. O. SelfAssembled Peptide Nanostructures for Functional Materials. Nanotechnology 2016, 27 (40), 402002.

(32) Morriss-Andrews, A.; Shea, J.-E. Simulations of Protein Aggregation: Insights from Atomistic and Coarse-Grained Models. J. Phys. Chem. Lett. 2014, 5 (11), 1899-1908.
(33) Manandhar, A.; Kang, M.; Chakraborty, K.; Tang, P. K.; Loverde, S. M. Molecular Simulations of Peptide Amphiphiles. Org. Biomol. Chem. 2017, 15 (38), 7993-8005.

(34) Moore, S.; Sonar, K.; Bharadwaj, P.; Deplazes, E.; Mancera, R. Characterisation of the Structure and Oligomerisation of Islet Amyloid Polypeptides (IAPP): A Review of Molecular Dynamics Simulation Studies. Molecules 2018, 23 (9), 2142.

(35) Shea, J.-E.; Urbanc, B. Insights into A $\beta$ Aggregation: A Molecular Dynamics Perspective. Curr. Top. Med. Chem. 2013, 12 (22), 2596-2610.

(36) Redler, R. L.; Shirvanyants, D.; Dagliyan, O.; Ding, F.; Kim, D. N.; Kota, P.; Proctor, E. a.; Ramachandran, S.; Tandon, A.; Dokholyan, N. V. Computational Approaches to Understanding Protein Aggregation in Neurodegeneration. J. Mol. Cell Biol. 2014, 6 (2), 104-115.

(37) Ning, L.; Guo, J.; Bai, Q.; Jin, N.; Liu, H.; Yao, X. Structural Diversity and Initial Oligomerization of PrP106-126 Studied by Replica-Exchange and Conventional Molecular Dynamics Simulations. PLoS One 2014, 9 (2), No. e87266.

(38) Gee, J.; Shell, M. S. Two-Dimensional Replica Exchange Approach for Peptide-Peptide Interactions. J. Chem. Phys. 2011, 134 (6), 064112.

(39) Mousseau, N.; Derreumaux, P. Exploring the Early Steps of Amyloid Peptide Aggregation by Computers. Acc. Chem. Res. 2005, 38 (11), 885-891.

(40) Liu, D.; Liu, F.; Zhou, W.; Chen, F.; Wei, J. Molecular Dynamics Simulation of Self-Assembly and Viscosity Behavior of PAM and CTAC in Salt-Added Solutions. J. Mol. Liq. 2018, 268, 131-139.

(41) Sasselli, I. R.; Moreira, I. P.; Ulijn, R. V.; Tuttle, T. Molecular Dynamics Simulations Reveal Disruptive Self-Assembly in Dynamic Peptide Libraries. Org. Biomol. Chem. 2017, 15 (31), 6541-6547.

(42) Lee, O.-S.; Cho, V.; Schatz, G. C. Modeling the Self-Assembly of Peptide Amphiphiles into Fibers Using Coarse-Grained Molecular Dynamics. Nano Lett. 2012, 12 (9), 4907-4913.

(43) Marrink, S. J.; Tieleman, D. P. Perspective on the Martini Model. Chem. Soc. Rev. 2013, 42 (16), 6801-6822.

(44) Gautieri, A.; Milani, A.; Pizzi, A.; Rigoldi, F.; Redaelli, A.; Metrangolo, P. Molecular Dynamics Investigation of Halogenated Amyloidogenic Peptides. J. Mol. Model. 2019, 25 (5), 124.

(45) Rigoldi, F.; Metrangolo, P.; Redaelli, A.; Gautieri, A. Nanostructure and Stability of Calcitonin Amyloids. J. Biol. Chem. 2017, 292 (18), 7348-7357.

(46) Bertran, O.; Curcó, D.; Zanuy, D.; Alemán, C. Atomistic Organization and Characterization of Tube-like Assemblies Comprising Peptide-Polymer Conjugates: Computer Simulation Studies. Faraday Discuss. 2013, 166, 59.

(47) Vijayaraj, R.; Van Damme, S.; Bultinck, P.; Subramanian, V. Structure and Stability of Cyclic Peptide Based Nanotubes: A Molecular Dynamics Study of the Influence of Amino Acid Composition. Phys. Chem. Chem. Phys. 2012, 14 (43), 15135.

(48) Lee, O.-S.; Stupp, S. I.; Schatz, G. C. Atomistic Molecular Dynamics Simulations of Peptide Amphiphile Self-Assembly into Cylindrical Nanofibers. J. Am. Chem. Soc. 2011, 133 (10), 36773683.

(49) Kang, M.; Chakraborty, K.; Loverde, S. M. Molecular Dynamics Simulations of Supramolecular Anticancer Nanotubes. J. Chem. Inf. Model. 2018, 58 (6), 1164-1168.

(50) Lee, O.-S.; Stupp, S. I.; Schatz, G. C. Atomistic Molecular Dynamics Simulations of Peptide Amphiphile Self-Assembly into Cylindrical Nanofibers. J. Am. Chem. Soc. 2011, 133 (10), 36773683.

(51) Cormier, A. R.; Pang, X.; Zimmerman, M. I.; Zhou, H.-X.; Paravastu, A. K. Molecular Structure of RADA16-I Designer SelfAssembling Peptide Nanofibers. ACS Nano 2013, 7 (9), 7562-7572.

(52) Nagy-Smith, K.; Moore, E.; Schneider, J.; Tycko, R. Molecular Structure of Monomorphic Peptide Fibrils within a Kinetically Trapped Hydrogel Network. Proc. Natl. Acad. Sci. U. S. A. 2015, 112 (32), 9816-9821. 